\title{
New supply modulation optimization methodology for Concurrent Dual Band Envelope Tracking Power Amplifier
}

\author{
Yiqiao Lin, Haedong Jang, Christophe Quindroit, Naveen Naraharisetti, and Patrick Roblin \\ The Ohio State University, Dept. of Electrical \& Computer Engineering Columbus, OH 43210, USA
}

\begin{abstract}
In this paper, the design, implementation, and measurement results of a concurrent dual-band envelope tracking (ET) system is presented. A GaN power amplifier (PA) is designed for concurrent dual-band operation. The iso-gain shaping functions for envelope tracking are then extracted from the characterization of the $P A$ in each band. To investigate the envelope tracking performance under concurrent dual-band operation and establish the optimal dual-band shaping function, a new supply modulation scheme is proposed. An experimental test bench is also presented. The performances of the new schemes are evaluated with a dual-band signal based on a LTE signal with PAR of $10 \mathrm{~dB}$ centered at $1892.8 \mathrm{MHz}$ and a WCDMA signal with PAR of $5 \mathrm{~dB}$ centered at $2200 \mathrm{MHz}$. Experimental results show 57 $\%$ average drain efficiency for peak output power of $36.6 \mathrm{dBm}$ and $36.5 \mathrm{dBm}$.
\end{abstract}

Index Terms-Power amplifiers, Concurrent Dual-Band, Envelope Tracking.

\section{INTRODUCTION}

The ever present demand for multi standard and multi band compatible and cost-effective base stations drives the need for highly-efficient, multi band power amplifiers (PAs). A central concern of base station power amplifier design comes from the linearity and efficiency requirements of the transmitter, which translate into signal integrity and operation cost.

Today, modern wireless communication standards employ high data rate and non constant envelope schemes such as orthogonal frequency division multiplexing (OFDM) in long term-evolution (LTE) transmission, introducing high envelope peak to average ratio (PAR) to the signal resulting in highly inefficient PAs when using traditional fixed power supplies. As a result, the evolution of supply modulation schemes such as envelope tracking (ET) have gained interest in order to improve the PA efficiency under high PAR requirements.

In the previous literatures for single-band PAs, the supply voltage responds to the instantaneous envelope $\left(E_{\text {inst }}\right)$ of the single-band modulation signal where the shaping function is expressed as $f_{\text {shaping }}\left(E_{\text {inst }}\right)$. For dual-band operation, following the instantaneous envelope becomes unrealistic, as the speed of the supply modulator needs to be at least 5 times faster than the beating frequency defined as $\Delta f=f_{U}-f_{L}$ of the dual band signal centered at the frequencies $f_{L}$ and $f_{U}$ for the lower side band (LSB) and the upper side band (USB) respectively. Cidronali et al. [1] demonstrated a prototype of dual band ET PA, where the shaping function is evaluated independently and optimized to maximize the efficiency on each single band mode respectively. On the other hand, Gilbert et al. [2] proposed an algorithm to generate slew-rate limited envelope to overcome the speed requirement.

In this paper, we propose a concurrent dual-band ET system that utilizes a new dynamic supply shaping scheme, using a weighted combination of the average and peak envelope of the instantaneous dual-band input signal.

The paper is organized as follows. Section II presents the various $2 \mathrm{D}$ analytic shaping functions proposed for the dualband ET modulation methodology. Section III discusses the PA characterization. Section IV describes the experimental setup and illustrates the efficiency of the proposed method.

\section{Proposed Envelope Tracking Supply MODULATION}

\section{A. 2D Supply Modulation Technique}

Let us define $E_{L}$ and $E_{U}$ as the input envelope of the LSB and USB respectively, separated by the frequency $\Delta f$. When the PA is simultaneously operating in two bands, a natural choice for the supply voltage VDD is to select a power weighted average of the single-band shaping-functions:

$$
\begin{aligned}
V_{D D}^{2 D}\left(E_{L}, E_{U}\right)=\frac{\left|E_{L}\right|^{2}}{\left|E_{L}\right|^{2}+\left|E_{U}\right|^{2}} f_{\text {shaping } L}\left(E_{L}\right) \\
+\frac{\left|E_{U}\right|^{2}}{\left|E_{L}\right|^{2}+\left|E_{U}\right|^{2}} f_{\text {shaping } U}\left(E_{U}\right),
\end{aligned}
$$

where $f_{\text {shapingL }}\left(E_{L}\right)$ and $f_{\text {shaping }}\left(E_{U}\right)$ are the shaping functions selected for mapping the envelope and the supply voltage for the LSB and USB respectively.

B. Supply Modulation based on the Average and the Peak of the Instantaneous Dual-Band Envelope

The dual-band instantaneous envelope of the input signal is written in terms of the input LSB $x_{L}$ and USB $x_{U}$ signals as:

$$
E_{\text {inst }}(t)=\left|x_{L} e^{-i \pi \Delta f t}+x_{U} e^{i \pi \Delta f t}\right|,
$$

which reaches a peak value of

$$
E_{\text {peak }}(t)=\operatorname{Max}\left(E_{\text {inst }}\right)=\left|E_{L}\right|+\left|E_{U}\right|,
$$

with $E_{L}=\left|x_{L}\right|$ and $E_{U}=\left|x_{U}\right|$. The average amplitude of the instantaneous input envelope is defined as:

$$
E_{\text {avg }}(t)=\operatorname{RMS}\left(E_{\text {inst }}\right)=\sqrt{\left|E_{L}\right|^{2}+\left|E_{U}\right|^{2}} .
$$

Fig. 1 illustrates the time domain representation of the instantaneous $\left(E_{\text {inst }}\right)$, peak $\left(E_{\text {peak }}\right)$ and average $\left(E_{\text {avg }}\right)$ dualband envelopes for an LTE/WCDMA signal combination. We can observe that $E_{\text {peak }}$ (blue) and $E_{\text {avg }}$ (black) are definitely 


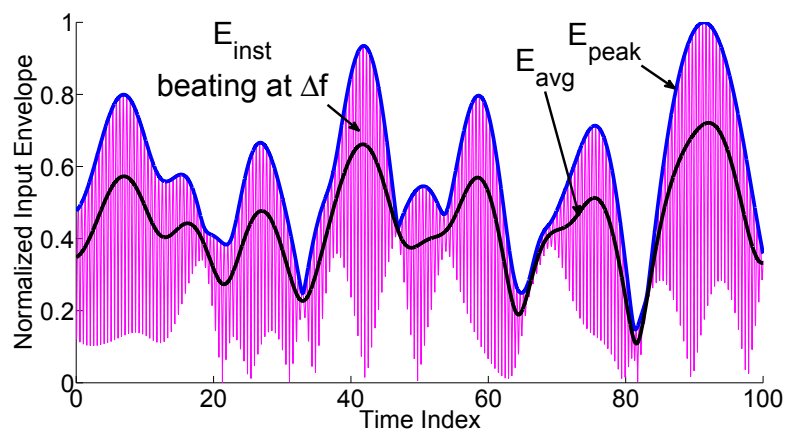

Fig. 1. Fast beating of the RF envelope (2) (purple line), compared to the peak envelope (3) (blue line), and average envelope (4) (black line).

varying more slowly than $E_{\text {inst }}$ (purple). Thus, the required supply voltage bandwidth is reduced.

We can select the modulated supply voltage to track $E_{\text {peak }}$ :

$$
\begin{aligned}
V_{D D}^{\text {peak }}\left(E_{L}, E_{U}\right)= & \frac{\left|E_{L}\right|^{2}}{\left|E_{L}\right|^{2}+\left|E_{U}\right|^{2}} f_{\text {shaping } L}\left(E_{\text {peak }}\right) \\
& +\frac{\left|E_{U}\right|^{2}}{\left|E_{L}\right|^{2}+\left|E_{U}\right|^{2}} f_{\text {shapingU }}\left(E_{\text {peak }}\right)
\end{aligned}
$$

where $f_{\text {shapingL }}$ and $f_{\text {shapingU }}$ are the shaping functions derived from the PA characterization results for each band. Alternatively, we can select the modulated supply voltage to track the average envelope $E_{\text {avg }}$ as:

$$
\begin{aligned}
V_{D D}^{a v g}\left(E_{L}, E_{U}\right)= & \frac{\left|E_{L}\right|^{2}}{\left|E_{L}\right|^{2}+\left|E_{U}\right|^{2}} f_{\text {shaping } L}\left(E_{\text {avg }}\right) \\
& +\frac{\left|E_{U}\right|^{2}}{\left|E_{L}\right|^{2}+\left|E_{U}\right|^{2}} f_{\text {shapingU }}\left(E_{\text {avg }}\right) .
\end{aligned}
$$

It has been observed that the saturation of a PA in dual-band operation ([3], Fig. 12) occurs when the normalized LSB $\left|y_{L}\right|$ and USB $\left|y_{U}\right|$ PA outputs are located between $\left|y_{L}\right|+\left|y_{U}\right|=1$ and $\left|y_{L}\right|^{2}+\left|y_{U}\right|^{2}=1$. Thus for a quasi-linear operation we can introduce the new envelope $E_{\epsilon}=\epsilon E_{\text {peak }}+(1-\epsilon) E_{\text {avg }}$ with a weighting factor $\epsilon$ between 0 and 1, to select an envelope between $E_{\text {peak }}$ and $E_{\text {avg }}$ :

$$
\begin{aligned}
V_{D D}^{\epsilon}\left(E_{L}, E_{U}\right)= & \frac{\left|E_{L}\right|^{2}}{\left|E_{L}\right|^{2}+\left|E_{U}\right|^{2}} f_{\text {shaping } L}\left(E_{\epsilon}\right) \\
& +\frac{\left|E_{U}\right|^{2}}{\left|E_{L}\right|^{2}+\left|E_{U}\right|^{2}} f_{\text {shapingU }}\left(E_{\epsilon}\right) .
\end{aligned}
$$

To determine $f_{\text {shapingL }}$ and $f_{\text {shaping }}$, a large-signal characterization using a continuous wave (CW) measurement will be separately conducted at both the 2 carrier frequencies.

\section{Dual-Band PA Characterization Results}

To verify the proposed concept, a dual-band power amplifier (Fig. 2) was designed and fabricated with a CREE GaN $15 \mathrm{~W}$ transistor CGH27015F [4]. The two bands are centered at $1892.8 \mathrm{MHz}$ and $2200 \mathrm{MHz}$ for the LSB and USB respectively. The large signal characterization is conducted using an LSNA by varying the supply voltage level VDD from $5 \mathrm{~V}$ to $25 \mathrm{~V}$.

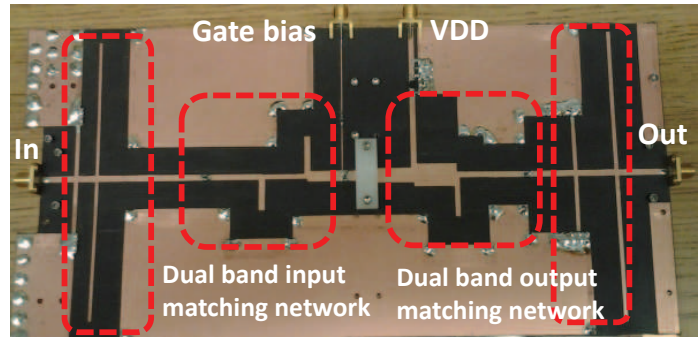

Fig. 2. PA designed for concurrent dual-band envelope tracking.

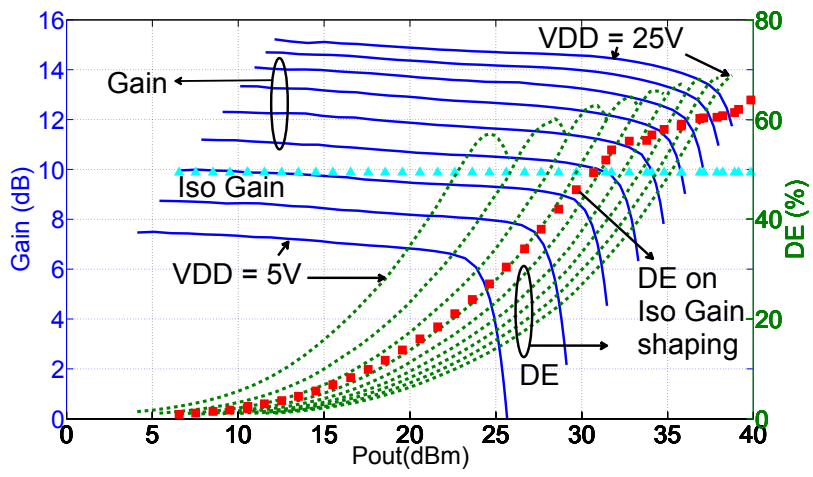

(a)

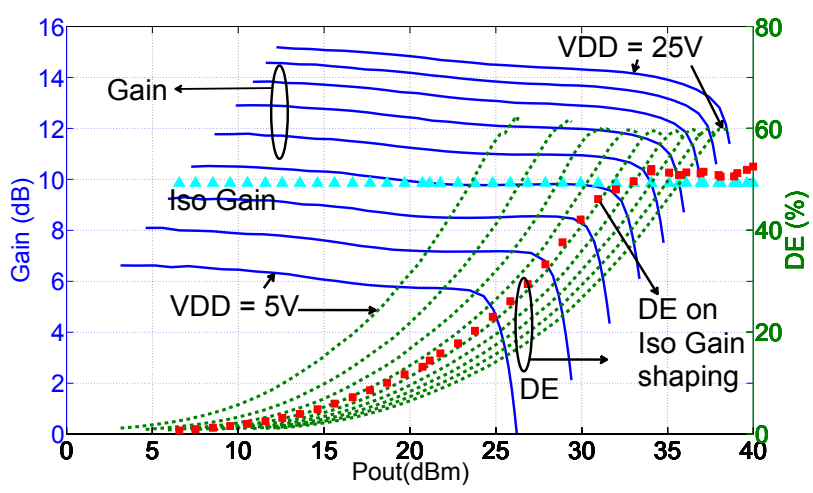

(b)

Fig. 3. Measured drain efficiency (DE) for $10 \mathrm{~dB}$ gain at $1.89 \mathrm{GHz}$ (a) and $2.20 \mathrm{GHz}$ (b) while VDD is varying from $5 \mathrm{~V}$ to $25 \mathrm{~V}$

As a result, the gain and the drain efficiency (DE) of the PA are measured. Fig. 3 reports the characterization result of the large signal measurements for both bands. At $1892.8 \mathrm{MHz}$, we observe that a peak drain efficiency of $68 \%$ at the peak output power of $38.7 \mathrm{dBm}$. At $2200 \mathrm{MHz}$, we notice a $60 \%$ peak drain efficiency at the peak output power of $38.5 \mathrm{dBm}$. The iso-gain ET shaping function (red squares) [5] is subsequently extracted by following the $10 \mathrm{~dB}$ gain line (cyan triangles).

\section{Concurrent Dual Band ET Experimental RESULTS}

\section{A. Dual Band ET System}

The envelope tracking setup is based on the test bed introduced in [6] and depicted in Fig. 4. It consists of two Analog 


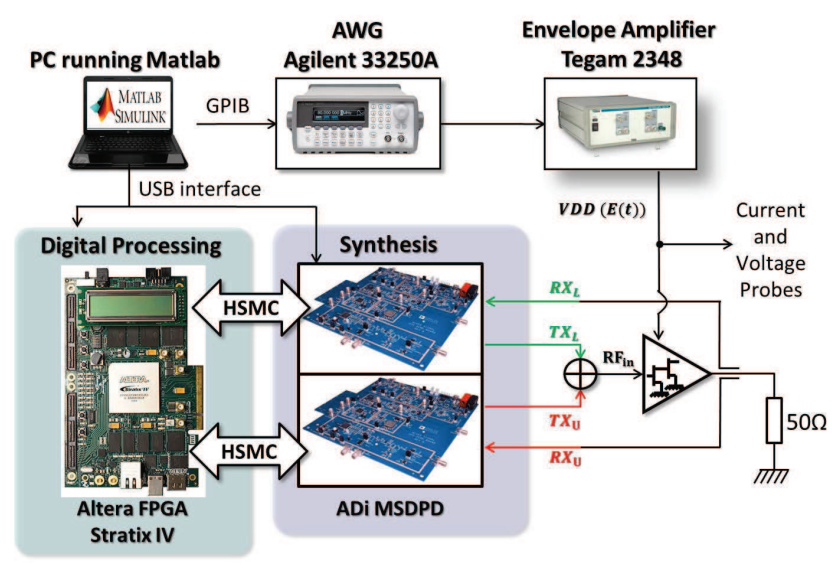

Fig. 4. Test setup for concurrent dual-band envelope tracking PA.

Devices Mixed Signal DPD (MSDPD) demo boards, both connected and clock synchronized to the Altera FPGA Stratix IV. The MSDPD enables the up/down conversion, filtering and DAC/ADC. The ADC sampling rate is $245.76 \mathrm{MHz}$. Both processed baseband signals are sent to their respective MSDPD to be up-converted to $1892.8 \mathrm{MHz}$ and $2200 \mathrm{MHz}$ for the lower side band (LSB) and upper side band (USB). Both generated $\mathrm{RF}$ signals are merged together to drive the amplification stage. The drain voltage signal is synthesized using Matlab and downloaded to the arbitrary waveform generator (AWG). The AWG is triggered by the FPGA in order to synchronize the drain voltage signal to the RF signal. The drain voltage signal is amplified by the TEGAM 2348 amplifier that is used as the supply modulator and drive the PA. Finally, the drain current and voltage are measured via two probes to calculate the drain efficiency.

\section{B. Envelope Tracking System Performances}

A LTE $5 \mathrm{MHz}$ signal with $10 \mathrm{~dB}$ PAR and a single carrier WCDMA signal with a $5 \mathrm{~dB}$ PAPR are used for the LSB and the USB respectively. The bandwidth of both baseband signals is reduced to suit the $2 \mathrm{MHz}$ cut-off frequency limitation of the TEGAM 2348 amplifier.

Table I shows the average dual-band output power and the average drain efficiency of the stand alone PA, for the 2D, average and peak envelope supply modulation techniques. For all scenarios, the peak output power reached is $36.6 \mathrm{dBm}$ and $36.5 \mathrm{dBm}$ for the LSB and USB respectively. We observe that the efficiency is improved from $41 \%$ with no ET system to more than $54 \%$ with the proposed ET schemes. Furthermore, the highest average drain efficiency of $57 \%$ is achieved for the average ET $(\epsilon=0)$. The lowest average drain efficiency of $54 \%$ is observed for the peak ET $(\epsilon=1)$.

The dual-band output power and drain efficiency obtained when varying the weighting factors $\epsilon$ is given in Fig. 5. It shows that the efficiency reduces monotonically when the envelope selected varies from the average ET $(\epsilon=0)$ to peak ET $(\epsilon=1)$ schemes.
TABLE I

DUAL-BAND AVERAGE OUTPUT POWER (DBM) AND DE (\%) FOR DIFFERENT SUPPLY MODULATION SCHEMES

\begin{tabular}{c||cccc}
\hline \hline & $\begin{array}{c}\text { Stand Alone PA } \\
\text { VDD }=25 \mathrm{~V}\end{array}$ & $\begin{array}{c}\text { 2D } \\
\text { Technique }\end{array}$ & $\begin{array}{c}\text { Average ET } \\
(\epsilon=0)\end{array}$ & $\begin{array}{c}\text { Peak ET } \\
(\epsilon=1)\end{array}$ \\
\hline Pout $(\mathrm{dBm})$ & 31.9 & 31.7 & 31.7 & 31.5 \\
DE $(\%)$ & 41.9 & 56.7 & 57.4 & 54.1 \\
\hline
\end{tabular}

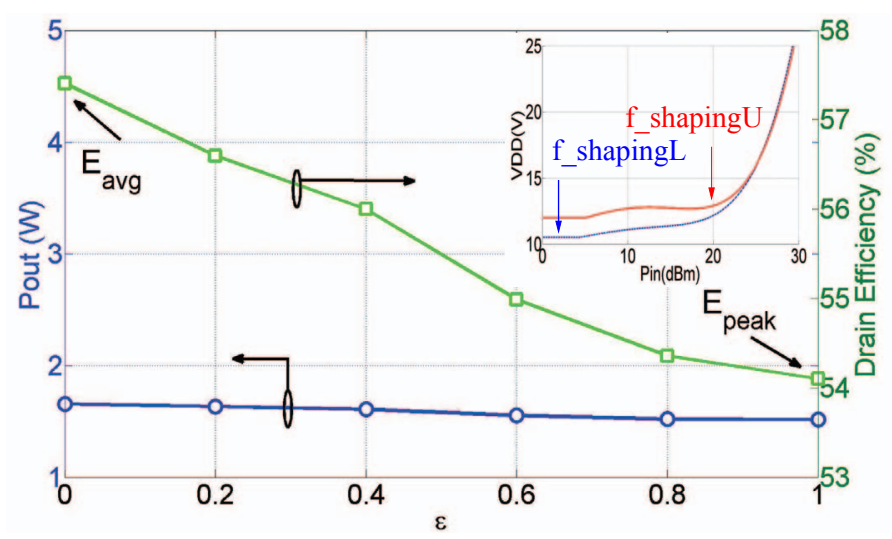

Fig. 5. Dual-band envelope tracking average output power and drain efficiency versus the weighting factor $\epsilon$.

\section{CONCLUSion}

This work proposed a new supply modulation scheme for dual-band envelope tracking power amplifier systems which relies on a weighted combination of the peak and average of the instantaneous envelopes. The experiments conducted compared the performance of the various shaping functions under concurrent dual-band envelope tracking operation. For the dual band power ET amplifier designed, the average envelope scheme exhibited the best efficiency. The prototype working at $1892.8 \mathrm{MHz}$ and $2200 \mathrm{MHz}$ reaches a peak output power of $36.6 \mathrm{dBm}$ and $36.5 \mathrm{dBm}$ and $57.4 \%$ average drain efficiency for a concurrent LTE/WCDMA dual-band signal.

\section{ACKNOWLEDGMENT}

The authors would like to thank Analog Devices Inc., Wilmington, MA for donating the Mixed Signal Digital Predistortion System Boards (MSDPD) which are used in this study. The authors would also like to thank Altera CorporationWireless Systems Solutions Group for the donation of the Stratix IV FPGA. This work was supported by the National Science Foundation under grant ECS 1129013.

\section{REFERENCES}

[1] A. Cidronali, N. Giovannelli, T. Vlasits, R. Hernaman, and G. Manes, "A $240 \mathrm{~W}$ dual-band 870 and $2140 \mathrm{MHz}$ envelope tracking GaN PA designed by a probability distribution conscious approach," in Microwave Symposium Digest (MTT), 2011 IEEE MTT-S International, June 2011, pp. $1-4$.

[2] P. Gilabert, G. Montoro, D. Lopez, and J. Garcia, "3d digital predistortion for dual-band envelope tracking power amplifiers," in Microwave Conference Proceedings (APMC), 2013 Asia-Pacific, Nov 2013, pp. 734-736.

[3] P. Roblin, C. Quindroit, N. Naraharisetti, S. Gheitanchi, and M. Fitton, "Concurrent linearization: The state of the art for modeling and linearization of multiband power amplifiers," IEEE Microwave Magazines, vol. 14, no. 7, pp. 75-91, Nov.-Dec. 2013. 
[4] CREE RF Products. [Online]. Available: www.cree.com/RF

[5] Understand and characterize envelope-tracking power amplifiers. [Online]. Available: http://www.nujira.com/ee-times-understand-andcharacterize-envelope-tracking-power-amplifiers-i-258.php

[6] C. Quindroit, N. Naraharisetti, P. Roblin, S. Gheitanchi, V. Mauer, and M. Fitton, "FPGA implementation of orthogonal 2d digital predistortion system for concurrent dual-band power amplifiers based on time-division multiplexing," Microwave Theory and Techniques, IEEE Transactions on, vol. 61, no. 12, pp. 4591-4599, Dec 2013. 